\title{
Playing off-line games with bounded rationality
}

\author{
Jérôme Renault* \\ CEREMADE \\ Université Paris Dauphine \\ Pl. du Marechal de Lattre de Tassigny \\ F-75775 Paris Cedex 16, France \\ renault@ceremade.dauphine.fr \\ Marco Scarsini \\ Dipartimento di Scienze Economiche e Aziendali \\ LUISS \\ Viale Pola 12 \\ I-00198 Roma, Italy \\ and HEC, Paris \\ mscarsini@luiss.it \\ Tristan Tomala \\ CEREMADE \\ Université Paris Dauphine \\ Pl. du Marechal de Lattre de Tassigny \\ F-75775 Paris Cedex 16, France \\ tomala@ceremade.dauphine.fr
}

November 21, 2007

\footnotetext{
* partly supported by the French Agence Nationale de la Recherche (ANR), undergrants ATLAS and Croyances, and the "Chaire de la Fondation du Risque", Dauphine-ENSAE-Groupama : Les Particuliers Face aux Risques.
} 


\begin{abstract}
We study a two-person zero-sum game where players simultaneously choose sequences of actions, and the overall payoff is the average of a one-shot payoff over the joint sequence. We consider the maxmin value of the game played in pure strategies by boundedly rational players and model bounded rationality by introducing complexity limitations. First we define the complexity of a sequence by its smallest period (a non-periodic sequence being of infinite complexity) and study the maxmin of the game where player 1 is restricted to strategies with complexity at most $n$ and player 2 is restricted to strategies with complexity at most $m$. We study the asymptotics of this value and a complete characterization in the matching pennies case. We extend the analysis of matching pennies to strategies with bounded recall.
\end{abstract}

Key words: Zero-sum games, periodic sequences, bounded recall, de Bruijn graphs, oblivious strategy.

MSC 2000 Subject Classification: Primary 91A05. Secondary 91A26.

OR/MS subject classification: Primary: Games/group decisions, noncooperative. 


\section{Introduction}

Dynamic decision theory models individual behavior by means of strategies, i.e. decision rules that adapt to the environment. In dynamic game models, the environment is potentially controlled by a rational agent which may display unpredictable behavior, out of strategic considerations. Therefore finding the optimal way to play or to respond to a complex behavior of the opponent might be difficult in terms of computation and costly to implement. One may thus want to study strategies that optimally plan the whole sequence of actions in a non-responsive way: these are called oblivious strategies, i.e. strategies that do not rely on observation. Oblivious strategies appear in a variety of contexts in operations research, see e.g. Alon et al. (2002), Chung et al. (2005), Dutta et al. (2002). This notion is also used in the literature on repeated games with bouded rationality, see e.g. O'Connell and Stearns (2003), Neyman and Okada (2005). Some papers study stochastic games played with oblivious strategies and the corresponding equilibria, see e.g. Weintraub et al. (2005).

The aim of the present paper is to study oblivious strategies per se in the simplest possible model of game. We consider a two-player zero-sum repeated game where each player chooses his whole sequence of actions beforehand. The payoff is an average of the sequence of payoffs generated by the joint sequence of actions. We call this the offline game. This is an extreme case of repeated game with imperfect monitoring where players have no observation at all. We investigate this game played by boundedly rational players.

The common assumption of perfectly rational agents has been questioned by several papers in game theory and a whole literature was born, where players are subject to some constraint in their ability to compute or to remember. Therefore only strategies that are not computationally too demanding are available to them. Bounds on players' rationality can be expressed in different forms. For instance one stream of literature considers games played by finite automata (see, e.g., Neyman (1985, 1998); Rubinstein (1986); Abreu and Rubinstein (1988); Kalai and Stanford (1988); BenPorath (1990, 1993); Neyman and Okada (2000b); Gossner and Hernández (2003, 2006); Gossner et al. (2003); Bavly and Neyman (2005)). A partially different stream of literature deals with games with bounded recall, i.e., games where players can remember only the most recent actions taken (see, e.g., Lehrer (1988, 1994); Sabourian (1998); Gossner et al. (2003); Bavly and Neyman (2005); Renault et al. (2006)). Other bounds on the complexity of the players have been considered, e.g., by Neyman and Okada $(1999,2000 a)$. This list is by no means exhaustive. Many of the existing papers consider zero-sum games and study the effect of different restrictions in the players' rationality on the outcome of the game. Our paper goes in this direction and studies two possible measures of complexity.

First we consider only periodic sequences and define the complexity of a sequence as the length of its smallest period (a non-periodic sequence is viewed as infinitely complex). This seems to be a natural notion of complexity to study as the usual 
ones, that is finite automata or bounded recall strategies, may only produce periodic sequences. Moreover, this corresponds to the size of the smallest automaton which is able to produce this sequence. To be more specific, a finite automaton (say for player 1) in this game is a tuple $\left(Q, q_{1}, f, h\right)$ where $Q$ is a finite nonempty set of states, $q_{1} \in Q$ is an initial state, $f: Q \rightarrow \mathcal{A}$ is the action function and $h: Q \rightarrow Q$ is the transition function. The difference with standard finite automata is that the transition here depends only on the internal state of the automaton as it receives no input from the opponent. The automaton then generates a sequence of actions $\left(x_{t}\right)_{t \geq 1}$ :

$$
x_{1}=f\left(q_{1}\right), q_{2}=h\left(q_{1}\right), \ldots, q_{t+1}=h\left(q_{t}\right), x_{t+1}=f\left(q_{t+1}\right),
$$

and so on. Since the set of states is finite, the sequences of states and of actions generated by the automaton are eventually periodic (periodic from some stage on) with period of length no more than $|Q|$ (the cardinality of $Q$ ). In the off-line game with the limit of the mean payoff, the transient phase of the automaton is irrelevant: given an eventually periodic sequence of actions for player 1, modifying finitely many terms to make it periodic does not change the payoff. Moreover, any sequence of actions with period $n$ can be played by an automaton with $n$ states. Our model may thus be regarded as a special case of repeated game played by finite automata (with trivial monitoring).

We study the maxmin value $V_{n, m}$ of the off-line game played in pure strategies, where player 1 is restricted to strategies with complexity at most $n$ and player 2 to strategies with complexity at most $m$. Our main result states that if player 1 has complexity at most $2 m$ and player 2 has complexity at most $m$, then player 1 can guarantee the value in mixed strategies of the stage game, up to an error of order $1 / m$. When the stage game is "matching pennies", we give a characterization of this maxmin value as a function of the pair complexities $(n, m)$.

The second complexity measure we consider is the size of the recall. A sequence has recall $k$ if each term of the sequence is given by a fixed function of the $k$ previous terms. This model is much more complicated to analyze than the previous one and we give results for matching pennies only. Our main result in this part states that one extra unit of recall ensures player 1 to guarantee a payoff close to the value of the one-shot game (asymptotically when $k$ is large): the advantage of having one extra unit of memory does not vanish at the limit.

The proofs are based on some arithmetic arguments about periodic sequences. For the model with bounded recall we use in addition some results on de Bruijn graphs and sequences. These sequences have already appeared in some bounded rationality models (see, e.g., Challet and Marsili (2000); Piccione and Rubinstein (2003); Liaw and Liu (2005); Gossner and Hernández (2006); Renault et al. (2006))

The paper is organized as follows. Section 2 describes the model. Section 3 deals with games with periodic strategies. Section 4 studies games with bounded recall. 


\section{Off-line games}

We start with a finite zero-sum game $G=(\mathcal{A}, \mathcal{B}, g)$ where $\mathcal{A}, \mathcal{B}$ are nonempty finite sets and $g: \mathcal{A} \times \mathcal{B} \rightarrow \mathbb{R}$. Player 1 chooses $a \in \mathcal{A}$, player 2 chooses $b \in \mathcal{B}$ and the payoff $g(a, b)$ is paid by player 2 to player 1 .

In the associated off-line game $\Gamma$, player 1 chooses an $\mathcal{A}$-valued infinite sequence $x=\left(x_{i}\right)_{i \geq 1}$, player 2 chooses a $\mathcal{B}$-valued infinite sequence $y=\left(y_{i}\right)_{i \geq 1}$, and the associated payoff is

$$
\gamma(x, y)=\lim \frac{1}{t} \sum_{i=1}^{t} g\left(x_{i}, y_{i}\right),
$$

where lim denotes a Banach limit, i.e. a linear mapping on the set of bounded sequences such that liminf $\leq \lim \leq \lim$ sup. The use of a Banach limit (usual in repeated games) will be immaterial in most of the paper since we shall deal mostly with converging sequences.

We shall use the following notations throughout the paper. For a finite set $\mathcal{A}$, we let $\Delta(\mathcal{A})$ be the set of probability distributions on $\mathcal{A}$. We use the same symbol for the multilinear extension of $g$, i.e., given a two finite sets $\mathcal{A}, \mathcal{B}$, a function $g: \mathcal{A} \times \mathcal{B} \rightarrow \mathbb{R}$ and two distributions $\mu \in \Delta(\mathcal{A}), \nu \in \Delta(\mathcal{B})$, we define

$$
g(\mu, \nu)=\sum_{a} \sum_{b} \mu(a) \nu(b) g(a, b)
$$

and we shall identify the degenerate distribution at a point $x$ with the point $x$ itself. We denote by $\operatorname{val}(G)$ the value of the game $G=(\mathcal{A}, \mathcal{B}, g)$ in mixed strategies,

$$
\operatorname{val}(G)=\max _{\mu \in \Delta(\mathcal{A})} \min _{b \in \mathcal{B}} g(\mu, b)=\min _{\nu \in \Delta(\mathcal{B})} \max _{a \in \mathcal{A}} g(a, \nu),
$$

by $\underline{v}(G)$ the maxmin in pure strategies,

$$
\underline{v}(G)=\max _{a \in \mathcal{A}} \min _{b \in \mathcal{B}} g(a, b),
$$

and by $\bar{v}(G)$ the minmax in pure strategies,

$$
\bar{v}(G)=\min _{b \in \mathcal{B}} \max _{a \in \mathcal{A}} g(a, b) .
$$

For a nonempty finite set $\mathcal{A}$, the set of $\mathcal{A}$-valued sequences is denoted $\mathcal{A}^{\omega}$. A sequence $x=\left(x_{t}\right)_{t \geq 1}$ is $n$-periodic if $x_{t+n}=x_{t}$ for each $t$. A sequence is periodic if it is $n$-periodic for some $n \geq 1$. The set of all periodic sequences is denoted by $S(\mathcal{A})$. For each $x$ in $S(\mathcal{A})$, we let $\operatorname{per}(x)$ be the smallest $n$ such that $x$ is $n$-periodic. For each $n \geq 1$, we let $S_{n}(\mathcal{A})$ be the set of periodic sequences $x$ such that $\operatorname{per}(x) \leq n$, and we let $S_{n}^{\prime}(\mathcal{A})$ be the set of $n$-periodic sequences, i.e. all sequences $x$ such that $\operatorname{per}(x)$ divides $n$. 
Let $\Delta_{n}(\mathcal{A})$ be the set of probability distributions which are fractional in $n$, that is $\mu \in \Delta_{n}(\mathcal{A})$ if for every $a \in \mathcal{A}$, the value $n \mu(a)$ is an integer (with an abuse of notation we write $\mu(a)$ instead of $\mu(\{a\}))$. An $n$-periodic sequence $x$ induces an empirical distribution $\mu_{x} \in \Delta_{n}(\mathcal{A})$, defined by:

$$
\mu_{x}(a)=\frac{1}{n} \sum_{i=1}^{n} \mathbf{1}_{\left\{x_{i}=a\right\}} .
$$

\section{Off-line games with periodic sequences}

The main goal of the paper is to study the maxmin in pure strategies of the off-line game when players are restricted to boundedly complex strategies. In this section we measure the complexity of a strategy in the off-line game, i.e. of a sequence, by its smallest period. A player with complexity $n$ and action set $\mathcal{A}$, may choose any periodic sequence of actions with period at most $n$, thus any sequence in $S_{n}(\mathcal{A})$. For $n, m \geq 1$ consider the quantity,

$$
V_{n, m}(G)=\max _{x \in S_{n}(\mathcal{A})} \min _{y \in S_{m}(\mathcal{B})} \gamma(x, y)
$$

which is the best payoff that player 1 can guarantee with a strategy of complexity at most $n$, against a strategy of player 2 with complexity at most $m$. From this definition, $V_{n, m}(G)$ is non-decreasing in $n$ and non-increasing in $m$.

\subsection{Maxmin values in the off-line game}

The off-line game $\Gamma$ has generally no value in pure strategies:

$$
\sup _{x \in \mathcal{A}^{\omega}} \inf _{y \in \mathcal{B}^{\omega}} \gamma(x, y)=\underline{v}(G)
$$

and,

$$
\inf _{y \in \mathcal{B}^{\omega}} \sup _{x \in \mathcal{A}^{\omega}} \gamma(x, y)=\bar{v}(G) .
$$

Indeed, if player 1 plays constantly an action $a$ that maximizes $\min _{b \in \mathcal{B}} g(a, b)$ over $\mathcal{A}$, one has $\gamma(x, y) \geq \underline{v}(G)$ for each $y$. On another hand, if we fix a sequence $x$ of player 1, player 2 may choose a sequence $y$ such that for each stage $t, y_{t}$ minimizes $g\left(x_{t}, b\right)$ over $b \in \mathcal{B}$. Thus, $\sup _{x \in \mathcal{A}^{\omega}} \inf _{y \in \mathcal{B}^{\omega}} \gamma(x, y) \leq \underline{v}(G)$.

Note that this implies that for each pair of complexities $(n, m), V_{n, m}(G) \geq \underline{v}(G)$ and if $n \leq m$, then $V_{n, m}(G)=\underline{v}(G)$.

The value of $\Gamma$ in mixed strategies clearly exists and equals val $(G)$. Player 1 (resp. player 2) guarantees $\operatorname{val}(G)$ by drawing an action at random according to an optimal mixed strategy in $G$ and playing constantly the selected action. 
Moreover, player 2 can defend the value of the game with constant strategies, i.e. with complexity one. Formally, for each $x \in \mathcal{A}^{\omega}$, there exists $y \in S_{1}(\mathcal{B})$ such that $\gamma(x, y) \leq \operatorname{val}(G)$. To see this, let $x \in \mathcal{A}^{\omega}$. For each $t \geq 1$ and $a \in \mathcal{A}$ define

$$
\mu_{x, t}(a)=\frac{1}{t} \sum_{i=1}^{t} \mathbf{1}_{\left\{x_{i}=a\right\}}
$$

the empirical distribution induced by $x$ up to stage $t$. For each $y \in S_{1}(\mathcal{B})$ constantly equal to $b$ we have

$$
\frac{1}{t} \sum_{i=1}^{t} g\left(x_{i}, y_{i}\right)=g\left(\mu_{x, t}, b\right)
$$

Let $\mu_{x}(a)=\lim _{t} \mu_{x, t}(a)$ the limit empirical distribution induced by $x$, where lim is a Banach limit (the usual limit may not always exist). Since lim is linear, this defines $\mu \in \Delta(\mathcal{A})$ such that $\gamma(x, y)=g(\mu, b)$. If player 2 chooses $b$ that minimizes $g(\mu, \cdot)$, then $\gamma(x, y) \leq \operatorname{val}(G)$.

Summing up, we get for each pair of complexities $(n, m)$ :

$$
\underline{v}(G) \leq V_{n, m}(G) \leq \operatorname{val}(G) \text {. }
$$

The next theorem states that the distance between $V_{2 m, m}(G)$ and $\operatorname{val}(G)$ is of order $1 / \mathrm{m}$. This shows that, to guarantee the fully rational solution of the game, here the value, player 1 needs only to be twice more complex than player 2 .

Theorem 3.1. For each $m \geq 2$,

$$
\operatorname{val}(G)-\frac{\|G\|}{m} \leq V_{2 m, m}(G) \leq \operatorname{val}(G),
$$

where $\|G\|:=\max _{b} \sum_{a}|g(a, b)|$.

The key to this theorem is to prove that when player 1 chooses a sequence with period $n$, player 2 has a best reply whose period divides $n$. In particular, when player 1 chooses a sequence with a prime period, the best that player 2 can do is to respond by a constant sequence. Hence, when player 2 has complexity $m$, player 1 with complexity $2 m$ may choose a prime period $p$ such that $m<p<2 m$ and choose a $p$-periodic sequence that guarantees the value of the stage game up to $1 / p \leq 1 / m$.

This method of proof suggests that Theorem 3.1 can by slightly improved as follows. For each integer $m$, let $p_{m}$ be the smallest prime number such that $p_{m}>m$, one has:

$$
\operatorname{val}(G)-\frac{\|G\|}{m} \leq V_{p_{m}, m}(G) \leq \operatorname{val}(G) .
$$

As $m<p_{m}<2 m$, this generalizes Theorem 3.1. However, $p_{m}$ is asymptotically smaller than $2 m$. 
We turn now to the formal proof of Theorem 3.1. We start by studying the problem of computing a best reply of player 2 within $S_{m}(\mathcal{B})$ against a periodic sequence $x \in$ $S(\mathcal{A})$. We fix a sequence $x$ with $\operatorname{per}(x)=n$, and an integer $m$. In the sequel we let $p=\operatorname{gcd}(n, m)$ be the greatest common divisor of $n$ and $m$, and $q=\operatorname{lcm}(n, m)$ be the least common multiple of $n$ and $m$. We consider the problem of finding an $m$-periodic, $\mathcal{B}$-valued sequence $y$ that minimizes the average of $g$ over a joint period of $(x, y)$, that is:

$$
\min _{y \in S_{m}^{\prime}(\mathcal{B})} \lim _{t} \frac{1}{t} \sum_{i=1}^{t} g\left(x_{i}, y_{i}\right)=\min _{y \in S_{m}^{\prime}(\mathcal{B})} \frac{1}{q} \sum_{i=1}^{q} g\left(x_{i}, y_{i}\right) .
$$

The $n$-periodic sequence $x$ is the repetition of a word of length $n$ with letters in $\mathcal{A}$, which is denoted $\tilde{x}=\left(x_{1}, \ldots, x_{n}\right)$. Likewise, we write $\tilde{y}=\left(y_{1}, \ldots, y_{m}\right)$. There are two integers $u, v$ such that $q=u n$ and $q=v m$, so that within a period of the bivariate sequence $(x, y), \tilde{x}$ is repeated $u$ times and $\tilde{y}$ is repeated $v$ times. For each $j \in\{1, \ldots, m\}$, we consider the $x_{i}$ 's that $y_{j}$ meets in the sequence $(x, y)$, that is we consider $\left\{x_{j+t m}: t \in \mathbb{N}\right\}$, and we look at the indices of these $x_{i}$ 's within a period of $x$.

For each integer $t \in \mathbb{N}$, let $[t]_{n}$ be the smallest positive element of the class of $t$ modulo $n$, that is $[t]_{n} \in\{1, \ldots, n\}$ and $t-[t]_{n}$ is a multiple of $n$. We let for each $j \in\{1, \ldots, m\}$,

$$
T_{n, m, j}=\left\{[j+t m]_{n}: t \in \mathbb{N}\right\} .
$$

We also denote $\mu_{x, m, j}$ the empirical distribution induced by the set of $x_{i}$ 's met by $y_{j}$, that is,

$$
\mu_{x, m, j}(a)=\frac{1}{\left|T_{n, m, j}\right|} \sum_{i \in T_{n, m, j}} \mathbf{1}_{\left\{x_{i}=a\right\}} .
$$

Hence, using notation (2.2),

$$
\frac{1}{q} \sum_{i=1}^{q} g\left(x_{i}, y_{i}\right)=\frac{1}{m} \sum_{j=1}^{m} g\left(\mu_{x, m, j}, y_{j}\right) .
$$

Therefore, to solve the minimization problem:

$$
\min _{y \in S_{m}^{\prime}(\mathcal{B})} \frac{1}{q} \sum_{i=1}^{q} g\left(x_{i}, y_{i}\right)
$$

one just has to choose $y_{j}$ that minimizes $g\left(\mu_{x, m, j}, b\right)$ over $b \in \mathcal{B}$.

Lemma 3.2. For every sequence $y \in S_{m}^{\prime}(\mathcal{B})$, and every pair of indices $j, j^{\prime} \in$ $\{1, \ldots, m\}$, we have:

$$
[j]_{p}=\left[j^{\prime}\right]_{p} \Longrightarrow T_{n, m, j}=T_{n, m, j^{\prime}}
$$

with $p=\operatorname{gcd}(n, m)$. 
Proof. Assume $[j]_{p}=\left[j^{\prime}\right]_{p}$, i.e. $j^{\prime}=j+k p$ for some integer $k$. Let $i \in T_{n, m, j^{\prime}}$. Then there exists two integers $s, t$ such that

$$
i=j^{\prime}+t m+s n=j+k p+t m+s n .
$$

From Bezout's identity (see e.g. Jones and Jones (1998)) there exist two integers $c, d$ such that $p=c n+d m$. It follows that

$$
i=j+(k c+s) n+(k d+t) m
$$

and thus $i \in T_{n, m, j}$. The conclusion is obtained by symmetry.

Lemma 3.2 shows that if two letters in $\tilde{y}$ have the same rank modulo $p=\operatorname{gcd}(n, m)$, then they meet the same set of letters of the sequence $x$. At optimum, these two letters can be chosen to be the same and thus $y$ can be chosen $p$-periodic.

Corollary 3.3. (a) For every $x \in S(\mathcal{A})$, the problem

$$
\min _{y \in S_{m}^{\prime}(\mathcal{B})} \lim _{t} \frac{1}{t} \sum_{i=1}^{t} g\left(x_{i}, y_{i}\right)
$$

has a solution $y$ such that $\operatorname{per}(y)$ divides $\operatorname{per}(x)$.

(b) For every $x \in S(\mathcal{A})$, the problem

$$
\min _{y \in S_{m}(\mathcal{B})} \lim _{t} \frac{1}{t} \sum_{i=1}^{t} g\left(x_{i}, y_{i}\right)
$$

has a solution $y$ such that $\operatorname{per}(y)$ divides $\operatorname{per}(x)$.

We may now prove Theorem 3.1.

Proof of Theorem 3.1. Given inequality (3.1), it is enough to prove that there exists $x \in S_{2 m}(\mathcal{A})$ such that, for each $y \in S_{m}(\mathcal{B})$,

$$
\gamma(x, y) \geq \operatorname{val}(G)-\frac{\|G\|}{m} .
$$

Bertrand's postulate, first proved by Chebyshev, states that for every integer $m \geq 2$, there exists a prime number $p$ such that $m<p<2 m$ (see e.g., Nagell (1964)).

Let $\mu$ be an optimal mixed strategy for player 1 in $G$. There exists $\mu^{p} \in \Delta_{p}(\mathcal{A})$ such that

$$
\left\|\mu-\mu^{p}\right\|_{\infty}:=\max _{a}\left|\mu(a)-\mu^{p}(a)\right| \leq \frac{1}{p} .
$$


The mapping $\mu \mapsto \min _{b} g(\mu, b)$ is $\|G\|$-Lipschitz, so that

$$
\min _{b} g\left(\mu^{p}, b\right) \geq \operatorname{val}(G)-\frac{\|G\|}{p} \geq \operatorname{val}(G)-\frac{\|G\|}{m} .
$$

There exists a sequence $x$ such that $\operatorname{per}(x)=p$ and $\mu_{x}=\mu^{p}$. To show this, it is enough to order the action set $\mathcal{A}=\left\{a_{1}, \ldots, a_{K}\right\}$ and play in sequence $a_{1}, p \mu^{p}\left(a_{1}\right)$ times, $\ldots, a_{K}, p \mu^{p}\left(a_{K}\right)$ times. From Corollary 3.3 , a best reply of player 2 , i.e. a sequence that achieves $\min _{y \in S_{m}(\mathcal{B})} \gamma(x, y)$, can be chosen such that $\operatorname{per}(y)$ divides $p$. As $p$ is prime, then $\operatorname{per}(y)=1$, that is $y$ is constant (say equal to $b$ ) and $\gamma(x, y)=$ $g\left(\mu^{p}, b\right)$. Thus, $\min _{y \in S_{m}(\mathcal{B})} \gamma(x, y)=\min _{b} g\left(\mu^{p}, b\right)$ which completes the proof.

We use now the previous construction to prove that a fully rational player 1 guarantees $\operatorname{val}(G)$ with a pure strategy against every periodic sequence of player 2 .

\section{Proposition 3.4.}

$$
\max _{x \in \mathcal{A}^{\omega}} \min _{y \in S(\mathcal{B})} \gamma(x, y)=\operatorname{val}(G)
$$

Proof of Proposition 3.4. We construct $x^{*} \in \mathcal{A}^{\omega}$ such that for each $y \in S(\mathcal{B})$,

$$
\gamma\left(x^{*}, y\right) \geq \operatorname{val}(G) \text {. }
$$

Let $\left(p_{n}\right)_{n}$ denote the sequence of prime numbers. For each $n$, denote by $x_{n} \in \mathcal{A}^{p_{n}}$ a word generating a sequence $x$ with smallest period $p_{n}$ and $\mu_{x}=\mu^{p_{n}}$ where $\mu^{p_{n}}$ is, as above, a $\left(1 / p_{n}\right)$-approximation of an optimal mixed strategy of player 1 . Such a word was just constructed in the proof of Theorem 3.1.

The sequence $x^{*}$ is constructed by concatenating those words. For all $n \geq 1$, call superword and denote $\tilde{x}_{n}$ the repetition of $x_{n},\left(p_{n}-1\right)$ ! times. Then $\tilde{x}_{n}$ has length $N_{n}:=\left(p_{n}\right)$ !. Choose then a sequence of integers $q_{n}$ such that:

$$
\frac{N_{n+1}}{q_{n} N_{n}} \rightarrow 0, \quad \text { as } n \rightarrow \infty \text {. }
$$

The sequence $x^{*}$ is such that $\tilde{x}_{1}$ is repeated $q_{1}$ times, $\tilde{x}_{2}$ is repeated $q_{2}$ times, $\ldots, \tilde{x}_{n}$ is repeated $q_{n}$ times, and so on.

Let $y \in S(\mathcal{B})$ and set $u=\operatorname{per}(y)$. For $n$ large enough, $p_{n}>u$, hence $u$ divides $p_{n}$ !. Since $\tilde{x}_{n}$ has length $p_{n}$ !, the periodic repetition of $\tilde{x}_{n}$ and $y$ have $p_{n}$ ! as common period. The average payoff over this period is thus the one yielded by $y$ and the periodic repetition of $\tilde{x}_{n}$. Denote this payoff $\gamma\left(\tilde{x}_{n}, y\right)$. As $p_{n}$ is prime and $p_{n}>u$, the best payoff that $y$ can achieve against $\tilde{x}_{n}$ is $\min _{b} g\left(\mu^{p_{n}}, b\right)$. Thus,

$$
\gamma\left(\tilde{x}_{n}, y\right) \geq \operatorname{val}(G)-\frac{\|G\|}{p_{n}} .
$$

Consider now $\gamma_{k}$ the average payoff yielded by the $k$-th superword against $y$. For each $k$, there is $n_{k}$ such that $\gamma_{k}=\gamma\left(\tilde{x}_{n_{k}}, y\right.$ ). (Since each superword is repeated many 
times, $n_{k}$ is only weakly increasing, e.g. for the first prime $p_{1}=2$, the superblock has two stages, thus $n_{1}=n_{2}=1$; for the second prime $p_{2}=3$, the superblock has 6 stages, thus $n_{3}=n_{4}=n_{4}=n_{6}=2$, and so on.) One has $\liminf \gamma_{k} \geq \operatorname{val}(G)$. Condition (3.3) ensures that the length of a superword is negligible with respect to the total length of what preceded it. Thus, the limit of the average payoff,

$$
\lim \frac{1}{t} \sum_{i=1}^{t} g\left(x_{i}, y_{i}\right)
$$

is the limit of a weighted average of the $\gamma_{k}$ 's, which yields $\gamma(x, y) \geq \operatorname{val}(G)$.

\subsection{The matching pennies game}

We give now a sharper result for the following matching pennies game, denoted $G^{*}$ in the sequel:

$$
\begin{array}{ll|l|}
\multicolumn{1}{l}{L} & \multicolumn{1}{l}{R} \\
\cline { 2 - 3 } T & 1 & 0 \\
B & 0 & 1 \\
\cline { 2 - 3 } & &
\end{array}
$$

The value of this game is $1 / 2$ and each player has a unique optimal mixed strategy which is $(1 / 2,1 / 2)$. We get here a characterization of $V_{n, m}\left(G^{*}\right)$. For every pair of integers $n, m$, let

$$
P(n, m)=\inf \left\{p \text { odd }: p \text { divides } n, \text { and } \frac{n}{p} \leq m\right\}
$$

and $P(n, m)=+\infty$ if there is no such $p$.

For instance, if $n \leq m$, then $P(n, m)=1$. If $m<n=2^{k}$, then $P(n, m)=+\infty$.

Theorem 3.5. For every pair of integers $n, m$,

$$
V_{n, m}\left(G^{*}\right)-\operatorname{val}\left(G^{*}\right)=\frac{-1}{2 \max _{k \leq n} P(k, m)} .
$$

Again we use the fact that when choosing his best reply, player 2 may choose a period that divides the period $n$ of player 1 . The key argument is as follows. Assume that $n=p q$ with $p$ odd, and that player 2 chooses a sequence with period $q$. Then each letter of player 2 faces a distribution of actions of player 1 which is fractional in $p$. Such a distribution must depart from the optimal strategy $(1 / 2,1 / 2)$ by at least $1 / 2 p$. We turn now to the formal proof.

Proof of Theorem 3.5. Here $\mathcal{A}=\{T, B\}$ and $\mathcal{B}=\{L, R\}$.

Clearly $S_{n}(\mathcal{A})=\cup_{k \leq n} S_{k}^{\prime}(\mathcal{A})$ and

$$
V_{n, m}\left(G^{*}\right)=\max _{k \leq n} \max _{x \in S_{k}^{\prime}(\mathcal{A})} \min _{y \in S_{m}(\mathcal{B})} \gamma(x, y) .
$$


We shall prove that

$$
w_{k, m}:=\max _{x \in S_{k}^{\prime}(\mathcal{A})} \min _{y \in S_{m}(\mathcal{B})} \gamma(x, y)=\frac{1}{2}-\frac{1}{2 P(k, m)} .
$$

If $k \leq m$, this formula holds, as $P(k, m)=1$ and $w_{k, m}=0$, since we can choose $y=x$. From now on, assume $k>m$. We consider two cases.

Case 1: $P(k, m)<+\infty$.

Set $p=P(k, m)$ so that $p$ is odd, divides $k$ and

$$
\ell:=\frac{k}{p} \leq m
$$

We first show that for every $x$ in $S_{k}^{\prime}(\mathcal{A})$, there exists $y$ in $S_{m}(\mathcal{B})$ such that

$$
\gamma(x, y) \leq \frac{1}{2}-\frac{1}{2 p}
$$

Let $x$ in $S_{k}^{\prime}(\mathcal{A})$ and let $y$ be an $\ell$-periodic sequence induced by the word $\tilde{y}=$ $\left(y_{1}, \ldots, y_{\ell}\right)$. The joint period of $(x, y)$ is then $k$, and the word $\tilde{y}$ is repeated $p$ times. Each $y_{j}$ meets thus $p$ letters $x_{i}$, and for each $j, \mu_{x, \ell, j} \in \Delta_{p}(\mathcal{A})$, where $\mu_{x, \ell, j}$ is defined as in (3.2). One can thus choose $\tilde{y}$ such that

$$
\gamma(x, y)=\frac{1}{\ell} \sum_{j=1}^{\ell} \min _{b \in \mathcal{B}} g\left(\mu_{x, \ell, j}, b\right) .
$$

A probability distribution $\mu \in \Delta_{p}(\mathcal{A})$ has the form

$$
\mu=(\mu(T), \mu(B))=\left(\frac{q}{p}, \frac{p-q}{p}\right)
$$

with $q \in\{0, \ldots, p\}$, and

$$
\min _{b \in \mathcal{B}} g(\mu, b)=\min (\mu(T), \mu(B))
$$

Since $p=2 d+1$ for some integer $d$, then for each $q \in\{0, \ldots, p\}$,

$$
\min \left(\frac{q}{p}, \frac{p-q}{p}\right) \leq \frac{d}{p}=\frac{1}{2}-\frac{1}{2 p}
$$

Therefore, for the chosen $y$, (3.5) holds.

We construct now $x \in S_{k}^{\prime}(\mathcal{A})$ such that for each $y \in S_{m}(\mathcal{B})$,

$$
\gamma(x, y) \geq \frac{1}{2}-\frac{1}{2 p}
$$


By definition $p$ is an odd divisor of $k$, and as $k>m, p>1$. We let $k=\ell p$ for some integer $\ell$, and $p=2 d+1$ with $d$ a positive integer. Let then $x$ be the periodic sequence generated by the word

$$
\tilde{x}=\left(x_{1}, \ldots, x_{k}\right)=\underbrace{T \ldots T}_{\ell d \text { times }} \underbrace{B \ldots B}_{\ell(d+1) \text { times }} .
$$

We claim that for this $x, \min _{y \in S_{m}(\mathcal{B})} \gamma(x, y)$ is achieved by the sequence which is constantly $L$ and thus,

$$
\min _{y \in S_{m}} \gamma(x, y)=\frac{\ell d}{k}=\frac{d}{p}
$$

Let $y$ that achieves this minimum. From Corollary 3.3, we may assume that $u:=$ $\operatorname{per}(y)$ divides $k$, so there is an integer $D$ such that $k=D u$. Since $u \leq m<k$, we have $D \geq 2$. Let $\left(y_{1}, \ldots, y_{u}\right)$ be the word generating $y$, we claim that each letter $y_{j}$ meets more $B$ 's than $T$ 's so at optimum, each $y_{j}$ must be chosen equal to $L$.

For each $j \in\{1, \ldots, u\}, y_{j}$ appears at stages $j+t u, t=0, \ldots, D-1$. We just need to check that less than half of these dates are before the time of the last $T$, i.e. we check that,

$$
|\{t=0, \ldots, D-1: j+t u \leq \ell d\}| \leq \frac{D}{2} .
$$

If $j+t u \leq \ell d$, then $t u<\ell d$. Thus,

$$
t<\frac{\ell d}{u}=\frac{D d}{p}=D \frac{d}{2 d+1}<\frac{D}{2},
$$

which completes the proof if $D$ is even.

If $D$ is odd, $D=2 c+1$ for some integer $c$ and the definition of $p$ implies $D \geq p$ (minimality). If $j+t u \leq \ell d$, then:

$$
t<D \frac{d}{2 d+1} \leq D \frac{c}{2 c+1}=\frac{D-1}{2}
$$

which completes the proof in this case.

Case 2: $P(k, m)=\infty$.

In this case $k$ has the form $k=p 2^{j}$ with $p$ odd and $j$ nonnegative integer such that $k / p=2^{j}>m$. We need to prove that $w_{k, m}=1 / 2$. As $w_{k, m} \leq 1 / 2$, we prove that there exists $x \in S_{k}^{\prime}(\mathcal{A})$ such that for each $y \in S_{m}(\mathcal{B}), \gamma(x, y) \geq 1 / 2$.

Consider then the sequence $x$ with $\operatorname{per}(x)=2^{j}$ induced by the word of length $2^{j}$

$$
\underbrace{T \ldots T}_{2^{j-1} \text { times }} \underbrace{B \ldots B}_{2^{j-1} \text { times }} \text {. }
$$

Given this sequence $x, \min _{y \in S_{m}(\mathcal{B})} \gamma(x, y)$ is achieved by $y$ such that $\operatorname{per}(y)$ divides $2^{j}$, and since $m<2^{j}, \operatorname{per}(y)=2^{\ell}$ with $\ell \leq j-1$. Thus the period of $y$ divides $2^{j-1}$, and each letter in $y$ meets as many $T$ 's and $B$ 's. Thus $\gamma(x, y)=1 / 2$. 
The following is obtained directly from Theorem 3.5.

Corollary 3.6. (a) If $n \leq m$, then $V_{n, m}\left(G^{*}\right)=0$.

(b) For each $N$ and $m<2^{N}, V_{2^{N}, m}\left(G^{*}\right)=1 / 2$.

(c) For each $m, V_{2 m, m}\left(G^{*}\right)=1 / 2$.

Proof. (a) If $n \leq m$, then for each $k \leq n, P(k, m)=1$. This follows thus from Theorem 3.5 .

(b) If $n=2^{N}$ for some $N$, then $n$ has no odd divisor other than 1 . Hence, for $m<n$, $P(n, m)=+\infty$ and $V_{n, m}\left(G^{*}\right)=1 / 2$.

(c) For each $m \geq 1$, there is a unique $N \geq 1$ such that $2^{N-1} \leq m<2^{N}$ and thus $m<2^{N} \leq 2 m$. Therefore, $\max _{k \leq 2 m} P(k, m)=+\infty$.

\section{Off-line games with bounded recall}

Another commonly used measure of complexity of strategies is the recall, that is the number of past values of the sequence on which the next value depends.

Definition 4.1. Given an nonempty finite set $\mathcal{A}$, a sequence $x \in \mathcal{A}^{\omega}$ has recall $k \in \mathbb{N}$ if there exists a mapping $f: \mathcal{A}^{k} \rightarrow \mathcal{A}$ such that for each $t>k, x_{t}=f\left(x_{t-1}, \ldots, x_{t-k}\right)$.

Such a sequence $x$ is eventually periodic. As for automata, the transient phase is irrelevant for our purposes, so we let $M_{k}(\mathcal{A})$ be the set of periodic sequences with recall $k$. For a sequence $x \in M_{k}(\mathcal{A})$, we have $\operatorname{per}(x) \leq|\mathcal{A}|^{k}$. However, there are sequences with period $|\mathcal{A}|^{k}$ which are not of recall $k$. Take for example the sequence

$$
\underbrace{T \ldots T}_{2^{k-1}} \underbrace{B \ldots B}_{\text {times } 2^{k-1} \text { times }} \text {. }
$$

Although of period $2^{k}$, this sequence does not have recall $k$, otherwise the $k$ last $T$ 's should be followed by a $T$ (assuming $2^{k-1}>k$ ).

In this section we study the maxmin value of the off-line game where players are restricted by the size of the recall

$$
W_{j, k}(G)=\max _{x \in M_{j}(\mathcal{A})} \min _{y \in M_{k}(\mathcal{B})} \gamma(x, y)
$$

\subsection{Matching pennies with bounded recall}

Since the analysis of off-line games with bounded recall is significantly more difficult, we concentrate on the matching pennies game $G^{*}$ defined in (3.4). The proofs of our result shall use the tools of the previous section and the theory of de Bruijn graphs (see, e.g., de Bruijn (1946) and Yoeli (1962) for some properties of these graphs). 
Definition 4.2. A directed graph $D_{k}$ called a de Bruijn graph if

- the set of vertices of $D_{k}$ is $\{T, B\}^{k}$,

- there is an edge from $x=\left(x_{1}, \ldots, x_{k}\right)$ to $y=\left(y_{1}, \ldots, y_{k}\right)$ if and only if $\left(x_{2}, \ldots, x_{k}\right)=\left(y_{1}, \ldots, y_{k-1}\right)$.

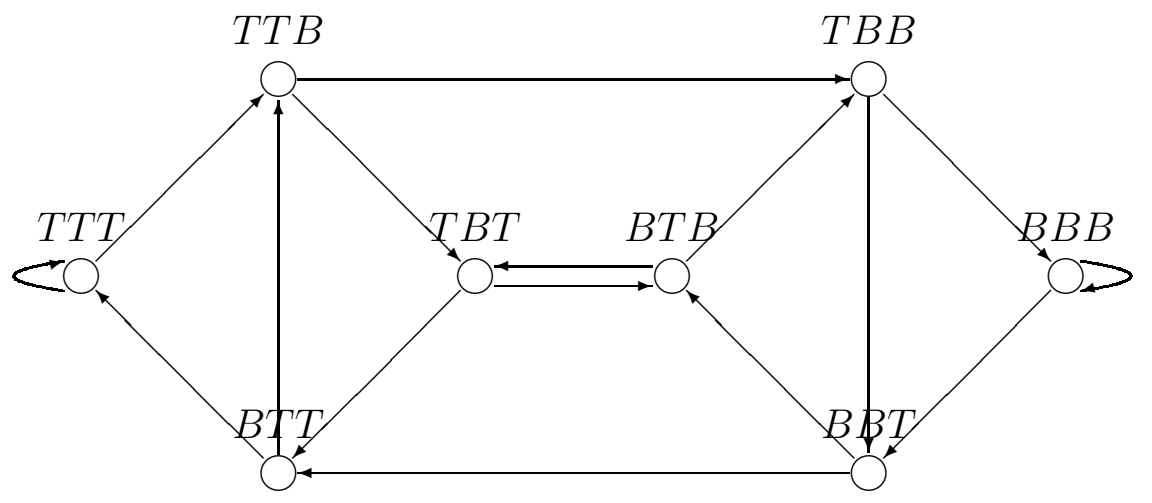

Figure 1. de Bruijn graph $D_{3}$

Consider player 1 with recall $k$. The set of possible recalls for player 1 is $\{T, B\}^{k}$. If the recall is the word $x \in\{T, B\}^{k}$ at some stage, the recall at the next stage is obtained by deleting the first letter of $x$ and adding a new letter after $x$. If $x=\left(x_{1}, \ldots, x_{k}\right)$, the next recall is either $\left(x_{2}, \ldots, x_{k}, T\right)$ or $\left(x_{2}, \ldots, x_{k}, B\right)$. This defines a de Bruijn graph.

A sequence with recall $k$ (for player 1 ) can the be viewed as a cycle in the de Bruijn graph $D_{k}$. Since $D_{k}$ has $2^{k}$ vertices, the longest possible cycle has length $2^{k}$. Since each vertex has as many outgoing as ingoing edges, such a cycle, called Hamiltonian cycle, exists (see, e.g., Bollobás (1998)). The associated sequence of $T \mathrm{~s}$ an $B \mathrm{~s}$ is called a de Bruijn sequence. A cycle of length 1 also exists (associated to the constant sequence TTT . . ), but, more generally, the following proposition (Yoeli (1962)) states that every length cycle is possible (see Lempel (1971) for a generalization to any finite alphabet).

Proposition 4.3. For every $p$ in $\left\{1, \ldots, 2^{k}\right\}$, there exists a cycle with length $p$ in the de Bruijn graph $D_{k}$.

The next lemma provides results similar to those obtained for automata.

Lemma 4.4. (a) For every pair of integers $(j, k), 0 \leq W_{j, k}\left(G^{*}\right) \leq 1 / 2$.

(b) If $j \leq k, W_{j, k}\left(G^{*}\right)=0$. 
(c) For every $k, W_{2^{k}, k}\left(G^{*}\right)=1 / 2$.

In Corollary 3.6 (c) player 1 can induce a period whose maximum length is twice the maximum length of the period induced by player 2. In Lemma 4.4 (c) player 1's maximum possible period is of length $2^{\left(2^{k}\right)}$, which is exponentially larger than the length of player 2's maximum possible period $2^{k}$. Hence here, in accordance with known results on zero-sum games with bounded complexity (see Lehrer (1988), BenPorath (1993)), if player 1 is exponentially more complex than player 2 , then she behaves like a fully rational player.

Proof of Lemma 4.4. (a) This is similar to inequality (3.1). Player 1 guarantees 0 with the constant sequence $T$, thus with recall zero. Player $2 \operatorname{defends} \operatorname{val}\left(G^{*}\right)$ with a constant strategy, thus again with recall zero.

(b) If player 2 has the largest recall, he can use the same sequences as player 1 and can defend 0 at every stage.

(c) If $j=2^{k}$, player 1 can choose the $2^{k+1}$-periodic sequence $x$ whose cycle is

$$
\underbrace{T \ldots T}_{2^{k} \text { times }} \underbrace{B \ldots B}_{2^{k} \text { times }}
$$

Note that such $x$ has indeed recall $2^{k}$. Each sequence $y \in M_{k}$ has period $\operatorname{per}(y) \leq$ $2^{k}<\operatorname{per}(x)$. As in the proof of Case 2 of Theorem 3.5 (with $p=1$ ), for each such $y$, $\gamma(x, y)=1 / 2$.

The main concern of this section is the study of $W_{k+1, k}\left(G^{*}\right)$.

Theorem 4.5. (a) $W_{1,0}\left(G^{*}\right)=W_{2,1}\left(G^{*}\right)=1 / 2, W_{3,2}\left(G^{*}\right)=3 / 7$,

(b) $\lim _{k} W_{k+1, k}\left(G^{*}\right)=1 / 2$.

Point (a) may suggest that the sequence $W_{k+1, k}\left(G^{*}\right)$ decreases away from $1 / 2$, the intuition being that the advantage of having one extra slot of recall vanishes as $k$ grows. Point (b) shows that it is not so.

Piccione and Rubinstein (2003, Section 5, Footnote 5) noticed that if player 1 plays a de Bruijn sequence of recall $k+1$, then player 2 with recall $k$ must "have a frequency of mistakes of at least $1 /(2(k+1))$." This statement implies that

$$
W_{k+1, k}\left(G^{*}\right) \geq \frac{1}{2(k+1)} .
$$

Theorem 4.5 states that for large values of $k$, if player 1 has recall $k+1$ and player 2 has recall $k$, player 1 can induce a frequency of mistakes close to $1 / 2$.

Proof of Theorem 4.5. (a) Applying point c of Lemma 4.4 for $k=0$ and $k=1$ gives $W_{1,0}\left(G^{*}\right)=W_{2,1}\left(G^{*}\right)=1 / 2$. We prove now that $W_{3,2}\left(G^{*}\right)=3 / 7$. We first show that $W_{3,2}\left(G^{*}\right) \geq 3 / 7$. 
Let $x$ be the 3 -recall strategy for player 1 that plays the 7 -periodic sequence TTTBBTB TTTBBTB .... Any strategy $y$ with recall 2 for player 2 has a period $\operatorname{per}(y) \leq 4=2^{2}$. From Corollary 3.3, the best sequence for player 2 can be chosen with a period that divides 7 , thus with period 1 . As the proportions of $T$ 's and $B$ 's are respectively $4 / 7$ and $3 / 7$, the best payoff that player 2 can get is $3 / 7$.

We prove now $W_{3,2}\left(G^{*}\right) \leq 3 / 7$ by checking that for each 3-recall strategy $x$, there exists a 2-recall strategy $y$ such that $\gamma(x, y) \leq 3 / 7$. We discuss on $p=\operatorname{per}(x)$, the period of $x$.

If $p=1, p=2$ or $p=4, x$ is indeed a 2-recall sequence: if $p=1$ this is a constant sequence, if $p=2$, up to circular permuations this is the sequence TBTB $\cdots$, and if $p=4$ this is the sequence TTBBTTBB $\cdots$ (again up to circular permutations). Thus, in each of these case, there exists $y$ such that $\gamma(x, y)=0$.

If $p \in\{3,5,7\}$, as these are prime numbers, the best $y$ is constant (Corollary 3.3). By choosing the less frequent action, player 2 ensure that the payoff is no more that $\frac{1}{2}-\frac{1}{2 p} \leq 3 / 7$ for $p \in\{3,5,7\}$.

If $p=8$, up to circular permutations and symmetry between $T$ and $B$, there is only one 8 -periodic sequence with recall 3 which is the de Bruijn sequence,

\section{BBBT BTTT BBBTBTTT ...}

Then, player 2 with recall 2 may play the 4 -periodic sequence $L L R R L L R R \ldots$ The payoff is here $2 / 8=1 / 4<3 / 7$.

Lastly assume $p=6$. We first rule out the 6 -periodic sequences that display different numbers of $T$ 's and $B$ 's. Assume that the number of $B$ 's is greater than the number of $T$ 's and call $q$ this number. Then $q<3$ and player 2 by playing $R$ gets $\frac{q}{6}<3 / 7$. If the number of $T$ 's and $B$ 's are equal, we enumerate all possible sequences for player 1, i.e. we enumerate all periods of length 6 containing $3 T$ 's and $3 B$ 's. Assume w.l.o.g. that the first element of the period is $T$. The periods of player 1 are ordered lexicographically (with $T$ preceding $B$ ). Nine cases are possible (how to select 2 stages among 5 for the last $2 T$ ), ruling out the 2-periodic sequences. In each case a periodic sequence of player 2 with recall 2 that gives a payoff lower than $\frac{3}{7}$ is displayed.

\begin{tabular}{|c|c|c|c|c|c|}
\hline case number & 1 & 2 & 3 & 4 & 5 \\
\hline period P1 & $T T T B B B$ & $T T B T B B$ & $T T B B T B$ & $T T B B B T$ & $T B T T B B$ \\
\hline sequence P2 & $R L R L R L$ & $L R L R L R$ & $R L R L R L$ & $L R L R L R$ & $R L R L R L$ \\
\hline payoff & $2 / 6$ & $2 / 6$ & $2 / 6$ & $2 / 6$ & $2 / 6$ \\
\hline
\end{tabular}

\begin{tabular}{|c|c|c|c|c|}
\hline case number & 6 & 7 & 8 & 9 \\
\hline period P1 & TBTBBT & TBBTTB & TBBTBT & TBBBTT \\
\hline sequence P2 & RLRLRL & RLRLRL & LRLRLR & RLRLRL \\
\hline payoff & $2 / 6$ & $2 / 6$ & $2 / 6$ & $2 / 6$ \\
\hline
\end{tabular}

In each case, the payoff is $2 / 6<3 / 7$. 
(b) By Bertrand's postulate, for each $k$ there exists a prime number $p$ such that $2^{k}<p<2^{k+1}$. By Proposition 4.3, there exists a $p$-periodic sequence of $T$ 's and $B$ 's that corresponds to a strategy with recall $k+1$. This defines $x$ in $M_{k+1}(\mathcal{A})$. As $p$ is prime, the best sequence that player 2 may choose among $S_{2^{k}}(\mathcal{B})$ and thus among $M_{k}(\mathcal{B})$ is a constant sequence.

Let $T(x)$ and $B(x)$ be the respective numbers of $T$ 's and $B$ 's in a cycle of $x$, so that

$$
\mu_{x}=\left(\frac{T(x)}{p}, \frac{B(x)}{p}\right) .
$$

The best payoff that player 2 can get is

$$
\min \left(\frac{T(x)}{p}, \frac{B(x)}{p}\right)
$$

and we just need to check that it is close to $1 / 2$ when $k$ is large. We assume w.l.o.g. $T(x) \geq B(x)$ and evaluate $B(x)$.

For each $i \geq k+2$, denote by $u_{i}=\left(x_{i-1}, \ldots, x_{i-(k+1)}\right)$ the recall of player 1 before stage $i$, and denote by $B\left(u_{i}\right)=\left|\left\{j \in\{i-(k+1), \ldots, i-1\}, x_{j}=B\right\}\right|$ the number of $B$ 's appearing in $u_{i}$. The sequence $\left(u_{i}\right)_{i \geq k+2}$ is periodic with period $p$, and

$$
\frac{1}{p} B(x)=\frac{1}{p}\left|\left\{i \in\{1, \ldots, p\}, x_{i}=B\right\}\right|=\frac{1}{p}\left(\sum_{i=k+2}^{k+1+p} \frac{1}{k+1} B\left(u_{i}\right)\right) .
$$

The point is that $u_{k+2}, u_{k+3}, \ldots, u_{p+k+1}$ are distinct elements of $\{T, B\}^{k+1}$, and $p>2^{k}$, so more than half of the words in $\{T, B\}^{k+1}$ appear in this average.

- Assume first $k$ even: $k=2 a$, with $a$ in $\mathbb{N}$. Then half of the words in $\{T, B\}^{k+1}$ contain more T's than $B$ 's, and we get a lower bound by selecting the $p$ elements with fewer $B$ 's. Better, we consider even less elements by taking average over the $2^{k}=2^{2 a}$ elements with less $B$ 's than T's. So

$$
\frac{B(x)}{p}>\frac{1}{2^{2 a}} \sum_{\ell=0}^{a} \frac{\ell}{2 a+1}\left(\begin{array}{c}
2 a+1 \\
\ell
\end{array}\right)=: F(a) .
$$

Since,

$$
\begin{aligned}
\sum_{\ell=0}^{a} \ell\left(\begin{array}{c}
2 a+1 \\
\ell
\end{array}\right) & =(2 a+1) \sum_{l=1}^{a} \frac{(2 a) !}{(\ell-1) !(2 a+1-\ell) !} \\
& =(2 a+1) \sum_{\ell=1}^{a}\left(\begin{array}{c}
2 a \\
\ell-1
\end{array}\right) \\
& =(2 a+1) \sum_{\ell=0}^{a-1}\left(\begin{array}{c}
2 a \\
\ell
\end{array}\right) \\
& =(2 a+1)\left(2^{2 a-1}-\frac{1}{2}\left(\begin{array}{c}
2 a \\
a
\end{array}\right)\right)
\end{aligned}
$$


then

$$
F(a)=1 / 2-\frac{1}{2^{2 a+1}}\left(\begin{array}{c}
2 a \\
a
\end{array}\right)
$$

So for $k$ even,

$$
\frac{B(x)}{p}>F(a)=1 / 2-\frac{1}{2^{2 a+1}}\left(\begin{array}{c}
2 a \\
a
\end{array}\right) .
$$

- Assume now that $k=2 a+1$ is odd. Proceeding the same way,

$$
\frac{B(x)}{p}>\frac{1}{2^{2 a+1}(2 a+2)}\left(\sum_{\ell=0}^{a} \ell\left(\begin{array}{c}
2 a+2 \\
\ell
\end{array}\right)+\frac{1}{2}(a+1)\left(\begin{array}{c}
2 a+2 \\
a+1
\end{array}\right)\right),
$$

and the right-hand side of this inequality is nothing but

$$
\frac{1}{2}+\frac{1}{2^{2 a+3}}\left(\begin{array}{c}
2 a+2 \\
a+1
\end{array}\right)-\frac{1}{2^{2 a+1}}\left(\begin{array}{c}
2 a+1 \\
a
\end{array}\right) .
$$

Hence,

$$
\frac{B(x)}{p} \geq \frac{1}{2}-\frac{1}{2^{2 a+1}}\left(\begin{array}{c}
2 a+1 \\
a
\end{array}\right) .
$$

The proof is completed by noticing that both

$$
\frac{1}{2^{2 a}}\left(\begin{array}{c}
2 a \\
a
\end{array}\right) \text { and } \frac{1}{2^{2 a+1}}\left(\begin{array}{c}
2 a+1 \\
a
\end{array}\right)
$$

go to zero as $a$ goes to infinity.

\subsection{Same recall, more actions}

To conclude the paper, we present an example showing that, in games with bounded recall, the complexity of the player may not be conveniently measured by the size of her recall. Consider the following game $G^{* *}$. It is a variation of matching pennies where each action of player 1 is duplicated.

\begin{tabular}{l|l|l|}
\multicolumn{1}{c}{} & \multicolumn{1}{c}{$L$} & \multicolumn{1}{c}{$R$} \\
\cline { 3 - 3 }$T_{1}$ & 1 & 0 \\
\cline { 2 - 3 }$T_{2}$ & 1 & 0 \\
\cline { 2 - 3 }$B_{1}$ & 0 & 1 \\
\cline { 2 - 3 }$B_{2}$ & 0 & 1 \\
\cline { 2 - 3 } & &
\end{tabular}

Proposition 4.6. $W_{k, k}\left(G^{* *}\right)=1 / 2$ for each $k \geq 1$.

Proof of Proposition 4.6. With recall $k$, player 1 can play the following $2^{k+1}$-periodic sequence: first play a de Bruijn sequence on the alphabet $\left\{T_{1}, T_{2}\right\}$ (of length $2^{k}$ ) followed by a de Bruijn sequence on the alphabet $\left\{B_{1}, B_{2}\right\}$. With recall $k$, player 2 cannot produce a period greater than $2^{k}$, and as the best reply has a period that divides $2^{k+1}$, player 2 cannot get more than $1 / 2$. 
Proposition 4.6 suggests that the actual power of player 1 with recall $k$ depends on her number of actions.

\section{Acknowledgment}

The work of Jerome Renault was partlysupported by the French Agence Nationale de la Recherche (ANR), undergrant ATLAS (JCJC06 137446).

The work of Marco Scarsini was partially supported by MIUR-COFIN. This paper was written while he was visiting CEREMADE, whose nice hospitality is gratefully acknowledged.

\section{References}

Abreu, D. and Rubinstein, A. (1988) The structure of Nash equilibrium in repeated games with finite automata. Econometrica 56, 1259-1281.

Alon, N., Guruswami, V., Kaufman, T., and Sudan, M. (2002) Guessing secrets efficiently via list decoding. In SODA '02: Proceedings of the thirteenth annual ACM-SIAM symposium on Discrete algorithms, 254-262. Society for Industrial and Applied Mathematics, Philadelphia, PA, USA.

Bavly, G. and Neyman, A. (2005) Online concealed correlation by boundedly rational players. Technical report, Center for the Study of Rationality, The Hebrew University of Jerusalem.

Ben-Porath, E. (1990) The complexity of computing a best response automaton in repeated games with mixed strategies. Games Econom. Behav. 2, 1-12.

Ben-Porath, E. (1993) Repeated games with finite automata. J. Econom. Theory 59, 17-32.

BollobÁs, B. (1998) Modern Graph Theory. Springer-Verlag, New York.

De Bruijn, N. G. (1946) A combinatorial problem. Nederl. Akad. Wetensch., Proc. 49, 758-764 = Indagationes Math. 8, 461-467 (1946).

Challet, D. and Marsili, M. (2000) Relevance of memory in minority games. Phys. Rev. E 62, 1862-1868.

Chung, F., Graham, R., Mao, J., and Yao, A. (2005) Oblivious and adaptive strategies for the majority and plurality problems. In Computing and combinatorics, volume 3595 of Lecture Notes in Comput. Sci., 329-338. Springer, Berlin. 
Dutta, D., Goel, A., and Heidemann, J. (2002) Oblivious AQM and Nash Equilibria. Technical Report 02-764, University of Southern California Computer Science Department. URL http://www.isi.edu/ johnh/PAPERS/Dutta02b.html.

Gossner, O. and Hernández, P. (2003) On the complexity of coordination. Math. Oper. Res. 28, 127-140.

Gossner, O. and Hernández, P. (2006) Coordination through De Bruijn sequences. Oper. Res. Lett. 34, 17-21.

Gossner, O., Hernández, P., and Neyman, A. (2003) Online matching pennies. Technical report, Center for the Study of Rationality, The Hebrew University of Jerusalem.

Jones, G. A. and Jones, J. M. (1998) Elementary Number Theory. Springer-Verlag London Ltd., London.

KAlai, E. and Stanford, W. (1988) Finite rationality and interpersonal complexity in repeated games. Econometrica 56, 397-410.

LEHRER, E. (1988) Repeated games with stationary bounded recall strategies. $J$. Econom. Theory 46, 130-144.

LEHRER, E. (1994) Finitely many players with bounded recall in infinitely repeated games. Games Econom. Behav. 7, 390-405.

Lempel, A. (1971) m-ary closed sequences. J. Combinatorial Theory Ser. A 10, $253-258$.

LiAw, S.-S. and LIU, C. (2005) The quasi-periodic time sequence of the population in minority game. Phys. A 351, 571-579.

Nagell, T. (1964) Introduction to Number Theory. Second edition. Chelsea Publishing Co., New York.

Neyman, A. (1985) Bounded complexity justifies cooperation in the finitely repeated prisoners' dilemma. Econom. Lett. 19, 227-229.

Neyman, A. (1998) Finitely repeated games with finite automata. Math. Oper. Res. 23, 513-552.

Neyman, A. and Okada, D. (1999) Strategic entropy and complexity in repeated games. Games Econom. Behav. 29, 191-223. Learning in games: a symposium in honor of David Blackwell.

Neyman, A. and Okada, D. (2000a) Repeated games with bounded entropy. Games Econom. Behav. 30, 228-247. 
Neyman, A. and OkadA, D. (2000b) Two-person repeated games with finite automata. Internat. J. Game Theory 29, 309-325.

Neyman, A. and OkadA, D. (2005) Growth of strategy sets, entropy, and nonstationary bounded recall. Technical Report 411, Center for the Study of Rationality, The Hebrew University of Jerusalem.

O'Connell, T. C. and Stearns, R. E. (2003) On finite strategy sets for finitely repeated zero-sum games. Games Econom. Behav. 43, 107-136.

Piccione, M. and Rubinstein, A. (2003) Modeling the economic interaction of agents with diverse abilities to recognize equilibrium patterns. J. European Econom. Assoc. 1, 212-223.

Renault, J., Scarsini, M., and Tomala, T. (2006) A minority game with bounded recall. Technical report, CEREMADE, Université de Paris Dauphine.

Rubinstein, A. (1986) Finite automata play the repeated prisoner's dilemma. J. Econom. Theory 39, 83-96.

Sabourian, H. (1998) Repeated games with $M$-period bounded memory (pure strategies). J. Math. Econom. 30, 1-35.

Weintraub, G. Y., Benkard, C. L., and Van Roy, B. (2005) Markov perfect industry dynamics with many firms. Technical Report W11900, NBER.

Yoeli, M. (1962) Binary ring sequences. Amer. Math. Monthly 69, 852-855.

November 21, 2007 